\title{
Quantitative evaluation of oligonucleotide surface concentrations using polymerization-based amplification
}

\author{
Ryan R. Hansen • Heather J. Avens • Raveesh Shenoy • \\ Christopher N. Bowman
}

Received: 30 April 2008 / Revised: 17 June 2008 / Accepted: 18 June 2008 / Published online: 26 July 2008

(C) The Author(s) 2008

\begin{abstract}
Quantitative evaluation of minimal polynucleotide concentrations has become a critical analysis among a myriad of applications found in molecular diagnostic technology. Development of high-throughput, nonenzymatic assays that are sensitive, quantitative and yet feasible for point-of-care testing are thus beneficial for routine implementation. Here, we develop a nonenzymatic method for quantifying surface concentrations of labeled DNA targets by coupling regulated amounts of polymer growth to complementary biomolecular binding on array-based biochips. Polymer film thickness measurements in the 20 $220 \mathrm{~nm}$ range vary logarithmically with labeled DNA surface concentrations over two orders of magnitude with a lower limit of quantitation at 60 molecules $/ \mu \mathrm{m}^{2}\left(\sim 10^{6}\right.$ target molecules). In an effort to develop this amplification method towards compatibility with fluorescence-based methods of characterization, incorporation of fluorescent nanoparticles into the polymer films is also evaluated. The resulting gains in fluorescent signal enable quantification using detection instrumentation amenable to point-of-care settings.
\end{abstract}

Keywords Signal amplification - Molecular diagnostics · Quantitative nucleic acid detection · DNA microarrays · Visible light photopolymerization · Surface-initiated polymerization

R. R. Hansen · H. J. Avens • R. Shenoy · C. N. Bowman $(\triangle)$ Department of Chemical and Biological Engineering, ECCH 111 CB 424, University of Colorado,

Boulder, CO 80309, USA

e-mail: Christopher.Bowman@Colorado.edu

\section{Introduction}

Quantitative evaluations of biomolecule concentrations are becoming increasingly necessary due to the expanding discovery and characterization of potential disease markers and the development of their corresponding applications in molecular diagnostics. Quantitative, sequence-specific analysis of polynucleotide biomarkers potentially allows for the monitoring of viral loads, allowing for drug efficacy evaluation $[1,2]$, elucidation of various disease states and progressions [3], and may provide clinical validation of a diagnosis [4]. Specifically with application to human cancers, several types of nucleic acid biomarkers require quantitative analysis, such as evaluation of over- or underexpressed RNA transcripts, analysis of genomic amplifications or deletions, as well as evaluation of epigenetic markers $[5,6]$. Mutation detection, traditionally performed on a qualitative basis, can also benefit from quantitative analysis through determination of wild-type to mutant-type ratios, as well as providing distinction between stochastic and pathogenic mutations [7].

Several challenges for quantitative biomolecular analysis in molecular diagnostics lie within the prerequisites of point-of-care implementation, requiring assays that are rapid and robust, use stable and inexpensive reagents, and are relatively simple to implement. The determination of sequence-specific nucleic acid concentrations has been developed through several PCR-based target amplification technologies, most notably real-time quantitative PCR [8]. Other technologies aim at achieving quantitative gains from signal amplification, such as rolling circle amplification [9], which has been creatively used in combination with BEAMing for sensitive quantitative detection [10], and branched DNA technology [11]. While such detection assays offer sensitive, powerful methods of quantitative 
amplification and detection, they are still generally limited by expensive and often complex protocols due to their reliance on enzymes and/or fluorescently labeled oligonucleotides for amplification, or may require instrumentation beyond the scope of point-of-care settings.

Recently, polymerization-based amplification has been demonstrated as a rapid, nonenzymatic method of signal amplification capable of offering visual detection of the presence of labeled polynucleotide targets on a biochip surface [12, 13]. With this method, amplification and detection are achieved by covalently coupling photoinitiator molecules to streptavidin proteins followed by binding onto biotinylated oligonucleotide targets immobilized to a biosensor surface. Upon contacting such biochips with monomer and light, a surface-initiated polymerization reaction occurring exclusively at the binding sites generates high molecular weight, highly visible polymer films that signal the binding event. While this method offers high amplification factors and sensitive, visual detection of biotinylated DNA molecules using assay conditions favorable for field implementation, analysis thus far is largely limited to a qualitative, positive/negative determination of the presence of model 3'-biotin-labeled capture sequences.

The purpose of this manuscript is to present a visible light polymerization-based amplification system that uses the biofunctional photoinitiator streptavidin-eosin isothiocyanate (SA-EITC) as a new method for quantifying surface concentrations of biotinylated nucleic acid targets. Such developments towards quantitation will allow for the implementation of this method in an increased number of molecular diagnostic applications. The potential for quantitation is investigated using array-based biochips containing spots with diluted amounts of 3'-biotin-labeled target oligos such that the amount of amplification as a function of oligo surface concentration can be determined. This approach of directly printing the biotinylated targets onto the surface serves as a model system [14] to evaluate the characteristics of polymerization-based amplification. Such a system is representative of a biochip after DNA target labeling with biotin that may be done either in solution [15] followed by hybridization to complementary capture probes, or through on-chip, primer extension labeling performed after complementary duplex formation [16-18]. Thus, the surface density of the biotinylated probe is proportional to the target solution concentration, dependent on the hybridization efficiency between a target and its surface-bound, complementary capture probe (typically, $K_{\mathrm{hyb}}=10^{9} \mathrm{M}^{-1}$ ) [19], and the labeling efficiency.

Incorporation of an amplified fluorescent, radiological, or chemiluminescent response with polymer film growth is also desirable due to the numerous types of inexpensive detection instrumentation consistent with these methods when signals are large. Fluorescence is particularly desir- able due to its sensitivity, safety, and multiplexing capabilities, and it is perhaps the most widely accepted method of detection, as evidenced by the wide number of amplification methods that aim at achieving large gains in fluorescent signal $[20,21]$. Due to these considerations, fluorescent moieties are added to monomer formulation to achieve an amplified, fluorescent signal that correlates with polymer growth. The amount of amplification from each binding site with known biotin capture probe density is then determined, either through direct measurement of film thickness or by measuring an amplified fluorescent signal, as shown in Fig. 1.

\section{Experimental}

\section{Materials}

Aminosilated glass slides were purchased from CEL Associates (Pearland, TX, USA). Streptavidin (SA) proteins were purchased from Pierce (Rockford, IL, USA) and stored at $4{ }^{\circ} \mathrm{C}$ until use. Polyethylene glycol diacrylate, $\mathrm{M}_{\mathrm{n}}=$ 575 Da (PEGDA), methyl diethanol amine (MDEA), 1vinyl-2-pyrrolidinone, dithiothreitol (DTT), 50x Denharts solution, and Tween 20 were purchased from Sigma (St. Louis, MO, USA). The fluorescent nanoparticles were $0.02 \mu \mathrm{m}$-diameter, carboxy-modified Nile Red FluoSpheres (Invitrogen, Carlsbad, CA, USA), encapsulating $\sim 10^{2}$ fluorophores per particle and supplied at a stock concentration of $4.5 \times 10^{15}$ nanoparticles $/ \mathrm{mL}$ containing $0.1 \%$ sodium azide preservative. The azide preservative was removed with the use of 100,000 Da dialysis columns (Spectra-Por, Rancho Dominguez, CA, USA). The purified FluoSpheres were stored at $4{ }^{\circ} \mathrm{C}$ until further use. Capture oligonucleotede sequences used were obtained from Operon (Huntsville, AL, USA) and were of the same sequence as previously reported [13].

\section{Dilution chip fabrication}

Dilution chips were fabricated according to a previous protocol [13]. Dilution chips contain fifteen sets of spots with different 3 ' biotin surface densities that span a range of three orders of magnitude $\left(10^{4}\right.$ labels $/ \mu \mathrm{m}^{2}$ to $<5$ labels $\left./ \mu \mathrm{m}^{2}\right)$. These chips were originally developed strictly to investigate sensitivity but were not sufficient for quantitative analysis. For this study, dilution chips were modified from their previous version by changing the spotting concentrations of several intermediate points. The resulting 3' biotin surface densities were determined using the calibration of spotting concentrations with surface densities that was previously developed [13]. In its current version, a more uniform distribution of biotin surface densities covering same range 


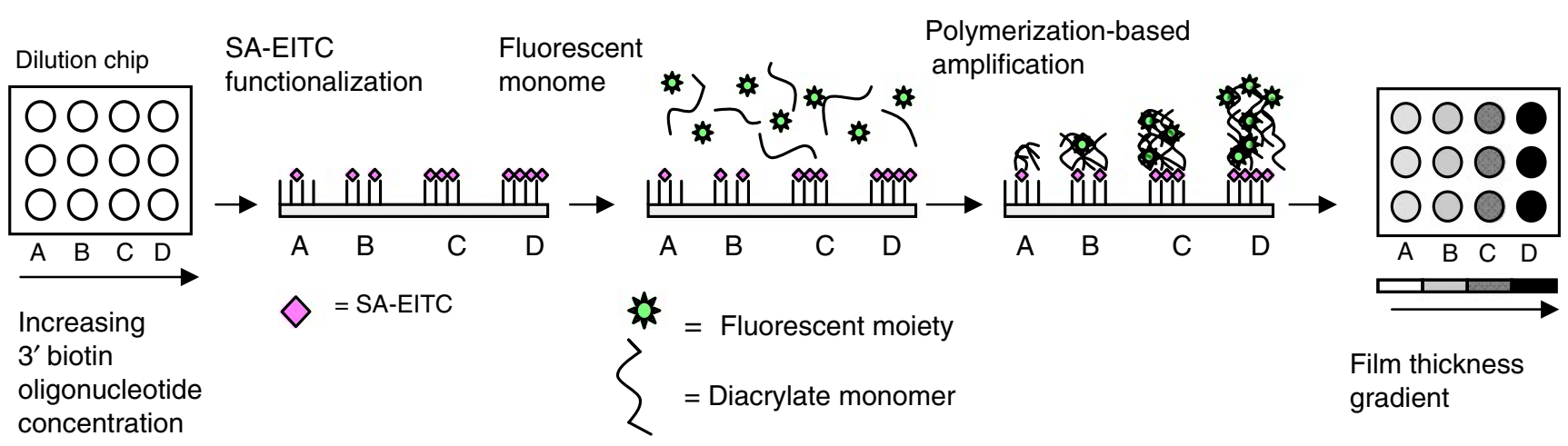

Fluorescence intensity gradient

Fig. 1 Dilution chips containing controlled amounts of 3 ' biotin oligonucleotides allow for an evaluation of the quantitative characteristics of polymerization-based amplification. Amplification is characterized by film thickness through the use of profilometry. In the case

is obtained within the array, allowing for a comprehensive evaluation of polymer growth with surface density. A sample layout of the new dilution chips and the corresponding 3' biotin surface densities is displayed in Fig. 2. Dilutions were spotted on aminosilated glass using a $375 \mu \mathrm{m}$ diameter stealth solid pin (ArrayIt, Sunnyvale, CA, USA) with a VersArray ChipWriter Pro system (Bio-Rad Laboratories, Hercules, CA, USA). Arrays were printed with replicate spots either within the same array or with replicate spots in an additional array on the same microarray slide.

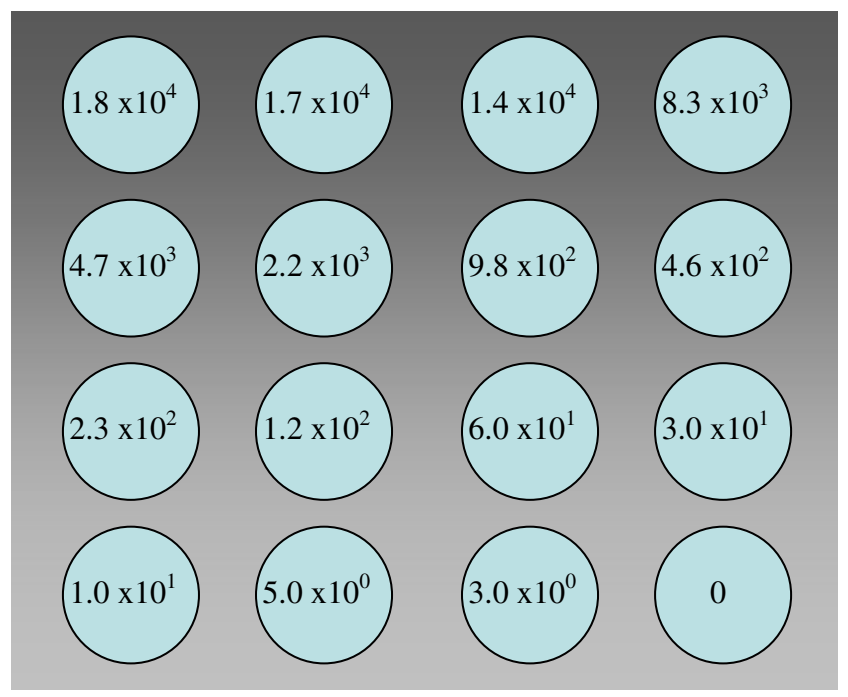

Fig. 2 Dilution chip layout with surface densities (molecules $/ \mu \mathrm{m}^{2}$ ) of 3'-biotin-labeled oligonucleotide capture probes on dilution chips. 3'biotin-labeled capture probes are diluted into a solution of unlabeled capture probes such that the overall surface density of capture oligo remained constant at $1.8 \times 10^{4} \mathrm{oligos} / \mu \mathrm{m}^{2}$ where fluorescent moiety is included in the monomer formulation, the extent of amplification can also be measured by the fluorescence intensity using a fluorescence microscope

Functionalization and characterization of dilution chips with SA-EITC

SA-EITC was synthesized, purified, and characterized according to a previously published protocol [13]. Dilution chips were functionalized with SA-EITC by applying a mixture of unlabeled SA and SA-EITC at $1 \mu \mathrm{g} / \mathrm{mL}$ in a $5 \mathrm{X}$ Denharts, 1 X PBS solution for 30 minutes followed by rinsing with wash buffer $(1 \mathrm{M} \mathrm{NaCl}, 0.1 \mathrm{M}$ Tris, $0.1 \%$ (wt/vol) Tween 20), then $\mathrm{H}_{2} \mathrm{O}$, and dried with $\mathrm{N}_{2}$. Preamplified, SA-EITC functionalized dilution chips were characterized using the green channel of a microarray scanner at $100 \%$ PMT gain (Agilent, Santa Clara, CA, USA) calibrated using a Cy3/Cy5 calibration chip (Full Moon Biosystems, Sunnyvale, CA, USA).

\section{Polymerization-based amplification}

Monomer solution contained $434 \mathrm{mM}$ PEGDA, $225 \mathrm{mM}$ MDEA, and $37 \mathrm{mM}$ 1-vinyl-2-pyrrolidinone in $\mathrm{H}_{2} \mathrm{O}$ (pH 9). Fluorescent monomer formulations included the addition of fluorescent nanoparticles to this monomer formulation at 90 or $500 \mathrm{nM}$ concentrations. Conventional UV-Vis spectroscopy was used to measure absorbance from fluorescent monomer formulations. Monomer was purged with argon and then $500 \mu \mathrm{L}$ was contacted with the SA-EITC functionalized dilution chip surface using a Whatman Chip Clip (Whatman, Florham Park, NJ, USA). Collimated, 495-650-nm polychromatic light was used to initiate polymerization from an Acticure light source (EFOS, Mississauga, ON, Canada) at an intensity of $10 \mathrm{~mW} / \mathrm{cm}^{2}$, as measured by an EFOS Cure-Rite radiometer. This was achieved with the use of an in-house 350-650-nm internal interference filter and a 490-nm stopband limit filter (Edmond 
Optics, Barrington, NJ, USA) applied to the end of a collimating lens attached to the end of a light guide. When fluorescent nanoparticles were included in monomer solutions, light intensity was increased accordingly to account for light attenuation occurring from the nanoparticles over a 1-mm-thick monomer layer. Prior to light exposure, the atmosphere was purged with argon for 5 min followed by light exposure for the desired time period. After amplification, unreacted monomer was removed from the surface by gently rinsing with water.

\section{Rhodamine B coupling into polymer films}

In an effort to achieve highly visible polymer films for visualization with fluorescence microscopy, methacryloxyethyl thiocarbamoyl rhodamine B (Polysciences, Warrington, PA, USA) was coupled to pendant double bonds present on PEG films on a surface through addition reactions with a DTT linker molecule. A $400 \mathrm{mM}$ solution of DTT was prepared in $0.1 \mathrm{M}$ carbonate buffer, $\mathrm{pH}$ 9.0. The DTT solution was contacted with the polymer chip for $25 \mathrm{~min}$, followed by $30 \mathrm{~min}$ of washing with $\mathrm{H}_{2} \mathrm{O}$. A solution of $4 \mu \mathrm{g} / \mathrm{mL}$ methacryloxyethyl thiocarbamoyl rhodamine $\mathrm{B}$ was then prepared in carbonate buffer and contacted with the DTT functionalized gel for $30 \mathrm{~min}$, followed by $30 \mathrm{~min}$ of washing in $\mathrm{H}_{2} \mathrm{O}$. This coupling procedure was useful as a method of visualizing polymer films with fluorescence microscopy, but was not sufficient for use in quantifying film thicknesses.

\section{Monitoring of bulk polymerization kinetics}

The initiating capability of SA-EITC in various monomer formulations was verified by monitoring bulk polymerization kinetics through the use of Fourier transform infrared (FTIR) spectroscopy (Nicolet Instrument Corporation, Madison, WI, USA). The conversion rate of carbon-carbon double bonds in monomer to carbon-carbon single bonds in polymer was measured by monitoring the absorbance at 6150 to $6212 \mathrm{~cm}^{-1}$ with time, using monomer formulations and light intensities identical to those used during on-chip polymerizations.

Post-amplification characterization of dilution chips

Film thicknesses were measured with a Dektak $6 \mathrm{M}$ profilometer (Veeco, Plainview, NY, USA) using a $12.5-\mu \mathrm{m}$ diamond stylus tip at a stylus force of $1 \mathrm{mg}$. Brightfield and fluorescent pictures of amplified dilution chips were obtained through the use of a Leica MZ FLIII stereomicroscope (Leica Microsystems, Wetzlar, Germany) calibrated with a Cy3/Cy5 calibration chip. Nile Red fluosphere-functionalized polymer films on the surface were further quantified with the use of an Agilent Technologies microarray scanner (green channel, PMT 1\%).

\section{Results and discussion}

Dependence of film thickness on surface oligonucleotide concentrations

The SA-EITC product has the ability to both initiate freeradical photopolymerizations by generating radicals through photoinduced electron transfer reactions with amine coinitiators, and to retain high-affinity binding to biotinylated substrates [13]. Upon the functionalization of a biotinylated dilution chip with SA-EITC, but before amplification, these dilution chips are imaged with a fluorescence scanner to verify the allocation of photoinitiators on the surface at densities proportional to the specific biotin label densities at each binding site. During amplification, long exposure times to low-intensity light were used to completely degrade SA-EITC, even from the spots containing the lowest initiator concentrations. This is followed by rapid propagation reactions of free radicals through monomer to generate high molecular weight polymer films on the surface. At long exposure times, long-wave visible light is desired to minimize or eliminate any nonspecific bulk polymerization that is characteristically observed from light sources with emissions in the UV region. Thus, polymerizations using polychromatic green light from a mercury arc lamp were performed. By examining several longer exposure times in the amplification, times of greater than $30 \mathrm{~min}$ were found to be required to obtain maximum sensitivity.

Upon amplification of this system at $30 \mathrm{~min}$, polymer films generated from as low as 60 biotin markers $/ \mu \mathrm{m}^{2}$ become visually observable under an optical microscope or to the unaided eye. Analysis of the film thicknesses at each site showed that a corresponding dynamic profile was achievable from the range of 60 to 4,700 biotin markers $/ \mu \mathrm{m}^{2}$. This was followed by a saturated region from 8,300 to 18,000 biotin markers $/ \mu^{2}$ with film thicknesses all in the $230 \pm$ $20 \mathrm{~nm}$ region, independent of marker density, as shown in Fig. 3. The dynamic region of amplification, approaching two orders of magnitude, fits a logarithmic correlation within error, which is typically less than or equal to $20 \%$. Further demonstrated in Fig. 3 is a tradeoff between assay time and sensitivity for this system. Exposing at times allowing for incomplete conversion of monomer into polymer has the ability to shift the sensitivity of the system, as $5 \mathrm{~min}$ of exposure shifts the sensitivity back to 2,200 biotin markers $/ \mu \mathrm{m}^{2}$. Such tunability allows the user to implement optimal assay conditions depending on the required sensitivity of the application. 
Fig. 3 a Fluorescent microscope image of a dilution chip amplified at $10 \mathrm{~mW} / \mathrm{cm}^{2}, 30$ min, followed by coupling rhodamine molecules to polymer films to enhance the visibility of the spots (reverse grayscale). The lowest concentration of targets that consistently produced polymer films with measurable thicknesses was 60 targets $/ \mu \mathrm{m}^{2}$ (circled in the figure). Lower concentrations produced visually detectable polymer films but were not measurable with profilometry. b Corresponding polymer film thicknesses versus labeled oligonucleotide surface density for two different exposure times

The increase in film thickness observed with higher biotin target concentrations is directly due to the corresponding increase of photoinitiator surface concentration on binding with SA-EITC. With a higher photoinitiator concentration on the surface, an increased number of propagating radical chains are generated during the polymerization process. Such free radicals are capable of diffusing from the surface and through the forming polymer film, thereby increasing the conversion of monomer into surface-bound polymer and ultimately increasing film thicknesses. This trend is consistent with both modeling and experimental findings of similar systems in the literature [22]. After the polymerization reaches high conversions, radical diffusivity throughout the hydrogel membrane decreases and eventually limits the extent of the polymerization. This results in the saturation of film thicknesses at spots containing higher concentrations of targets.

Competitive binding to tune regions of quantitative amplification

The dynamic dependence of the film thickness on photoinitiator surface concentration allows for an additional method of tuning the sensitivity and dynamic amplification of this assay to concentration regions of interest. By varying the binding parameters on the surface, allocation of different amounts of photoinitiator to sites containing a constant amount of biotin target is achievable. This potentially gives the user the ability to detect and quantify biotinylated DNA concentrations that may be either undetectable with less efficient binding or that may be in a saturated region with more efficient binding.

A simple demonstration of this technique can be accomplished through the use of a competitive binding technique involving incubating dilution chips with solutions containing various ratios of unmodified SA with SA-EITC. Due to the

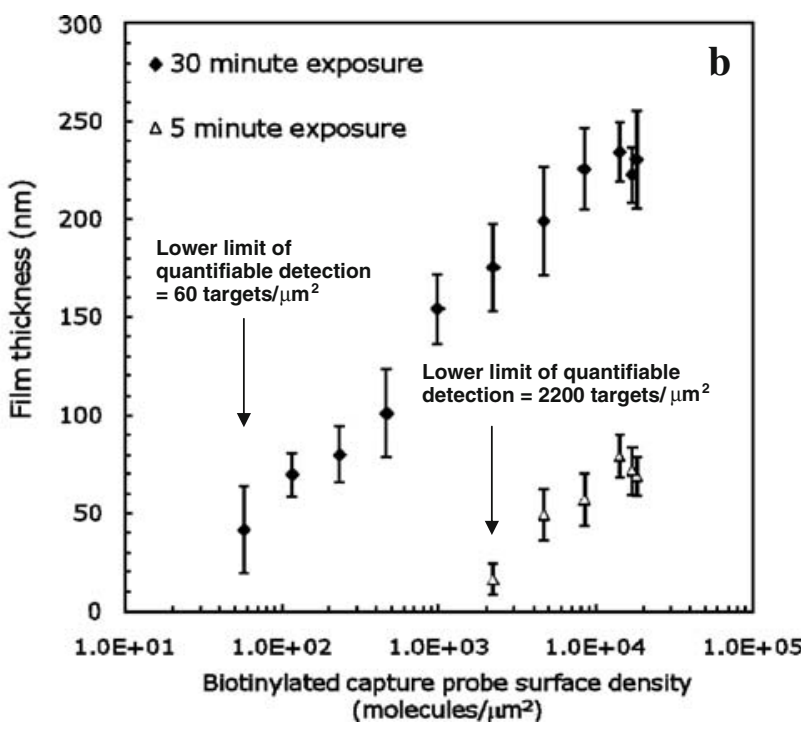

binding of unmodified SA to biotinylated DNA, the concentration of biotinylated DNA available for binding with SA-EITC decreases with higher SA to SA-EITC ratio. Because eosin fluoresces upon excitation with a $532 \mathrm{~nm}$ excitation source, a microarray scanner can be used to quantify the amount of SA-EITC binding on dilution chips incubated at various SA to SA-ETIC ratios. As shown in Fig. 4a, a shift towards lower fluorescent intensities and thus lower photoinitiator surface densities at identical biotin concentrations can be observed on dilution chips functionalized with higher SA:SA-EITC ratios. This enables higherend spots with photoinitiator concentrations corresponding to saturation from amplification without the addition of SA to shift to concentrations spread over the quantifiable region at higher SA:SA-EITC ratios. The result of this is a shift in detection limits towards less sensitive detection, but also a shift in the concentration regions of dynamic amplification, as shown in Fig. 4b. Competitive binding allows for quantification at higher-end concentrations ranging from $2,200-18,000$ biotins $/ \mu \mathrm{m}^{2}$ and extends the overall quantifiable regions from 60 to 18,000 biotins $/ \mu \mathrm{m}^{2}$. Assuming hybridization constants typical of complementary duplex formation on microarray surfaces $\left(K_{\mathrm{hyb}}=10^{9} \mathrm{M}^{-1}\right)$ [21] and that each target sequence can be labeled with a single biotin marker, the regions of quantification amplification correspond to analyte concentrations in the $\mathrm{nM}$ to $\mathrm{pM}$ range.

In the case of detecting nucleic acid hybridization with polymerization-based amplification, an alternative and perhaps more feasible approach involves varying capture sequence densities. The capture sequence density has been shown both experimentally and through modeling considerations to be a crucial parameter for achieving an optimal signal from hybridization [23, 24]. In an effort to shift a saturated response generated from complementary targetcapture hybridization to a quantifiable response, sites with 

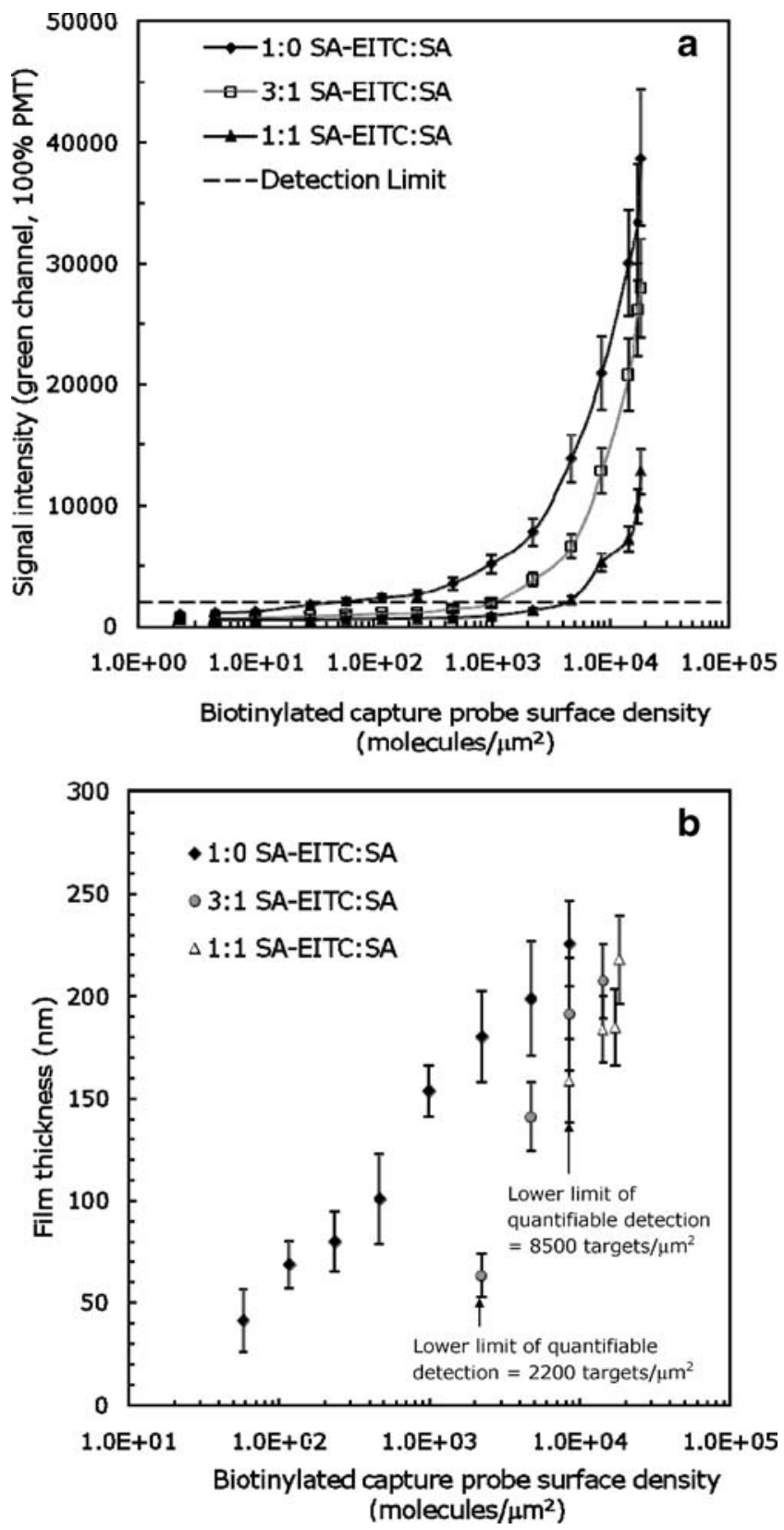

Fig. 4 a Fluorescent intensity profiles on dilution chips before amplification using competitive binding assay at various SA-EITC to SA ratios. b Corresponding film thicknesses from polymerizationbased amplification for each SA-EITC:SA ratio (30 min exposure). Points with saturated thicknesses are not shown on the plot

less then optimal capture probe densities could be printed. Such sites would then render lower photoinitiator concentrations than optimal sites at the same target concentrations, potentially shifting a saturated response to a quantifiable response. With the high-throughput capability of DNA chips, several sites of identical complementary capture sequences could be printed at optimal and successively less than optimal conditions within a single array, allowing for a quantifiable response to be achieved over many orders of magnitude.
Coupling amplified fluorescent signal with polymer growth

To implement inexpensive detection instrumentation for quantification, it is essential that PEG films with quantitative thicknesses generated from polymerization-based amplification be correlated with a measurable signal consistent with inexpensive methods of characterization. Direct film thickness measurements at the nanometer level are generally unfeasible in clinical settings due to the expense of the necessary instrumentation (profilometry, ellipsometry, atomic force microscopy). However, developing this amplification system in order to aim to generate an amplified fluorescent signal that corresponds with film thickness is particularly desirable, due to the numerous types of instrumentation available for characterizing biomolecule interactions using fluorescence measurements [25]. For array-based biosensors, such instrumentation ranges from microarray scanners using photomultiplier tube (PMT) detectors (detection limit $<0.05$ fluors $/ \mu \mathrm{m}^{2}, 10^{6}$ dynamic range) [26], costing hundreds of thousands of dollars, to inexpensive, portable fluorescence readers employing detection with less-sensitive complementary metal oxide semiconductor or charged coupled device (CCD) detectors that are more amenable to point-of-care testing. With these considerations in mind, an initial demonstration of the use of polymerization-based amplification to achieve an amplified, quantitative fluorescent signal is demonstrated herein.

The preferred approach to coupling fluorescent signal with polymer growth is to include the fluorescent moiety in the monomer formulation such that it is copolymerized or encapsulated into the crosslinked polymer network. With the commercial availability of several acrylated fluorophores, copolymerization of molecules such as fluorescien$o$-acrylate into PEG-based polymer films on a solid substrate has been demonstrated as a method of coupling a fluorescent response to hydrogel formation [27]. When applied to photopolymerization-based amplification, limitations to such approaches are inherent, namely: nonspecific initiation of the bulk monomer due to photoreduction reactions occurring between photoexcited fluorophores and amine coinitiators; decreased initiation rates from the surface-bound eosin initiators due to light attenuation to the surface caused by the absorbing fluorophore; and finally, saturation of the fluorescent signal after amplification due to the quenching of fluorescent molecules at higher concentrations. An alternative to fluorescent acrylates are commercially available 20 -nm-diameter polystyrene microspheres that encapsulate large numbers of fluorophores per particle. These fluorescent particles mitigate many of the above limitations, largely due to the fact that they minimize fluorophore contact with their surrounding environment and remain strongly fluorescent. 
To achieve fluorescent signal gains using polymerization-based amplification, 20-nm-diameter Nile Red (532/ 575) FluoSpheres $\left(\varepsilon_{532}=2.3 \times 10^{6} \mathrm{~cm}^{-1} \mathrm{M}^{-1}\right)$ were added to monomer solution at various concentrations. Figure 5 shows the absorbance spectra of the monomer solution containing $90 \mathrm{nM}$ fluorescent nanoparticles compared to that of a $10 \mu \mathrm{g} / \mathrm{mL}$ solution of SA-EITC. There is a considerable overlap in absorbance between the monomer solution and the initiator at wavelengths in the $500-650 \mathrm{~nm}$ region used to initiate the polymerization reaction. Thus, adsorption of light from fluorescent monomer effectively decreases initiation rates from SA-EITC at constant light intensity. This effect is observed when monitoring bulk polymerization kinetics under initiation conditions similar to those used during on-chip amplification. The monomer conversion into polymer with time is shown in Fig. 6 using $85 \mu \mathrm{g} / \mathrm{mL}$ SA-EITC (equivalent to $2 \mu \mathrm{M}$ eosin), a concentration representing the local concentrations of SA-EITC typically obtained over positive spots on the dilution chips containing dilute amounts of biotin. Both the polymerization kinetics and the final conversion of monomer to polymer decrease due to the addition of nanoparticles. The decrease in final conversion suggests that nanoparticles may be capable of terminating propagating free radicals during the polymerization process.

An important observation from Fig. 6 is that without the addition of SA-EITC to monomer, polymerization is only observed at long exposure times (greater than $40 \mathrm{~min}$, shown in curve d of Fig. 6). Photoinitiation due to photoexcited

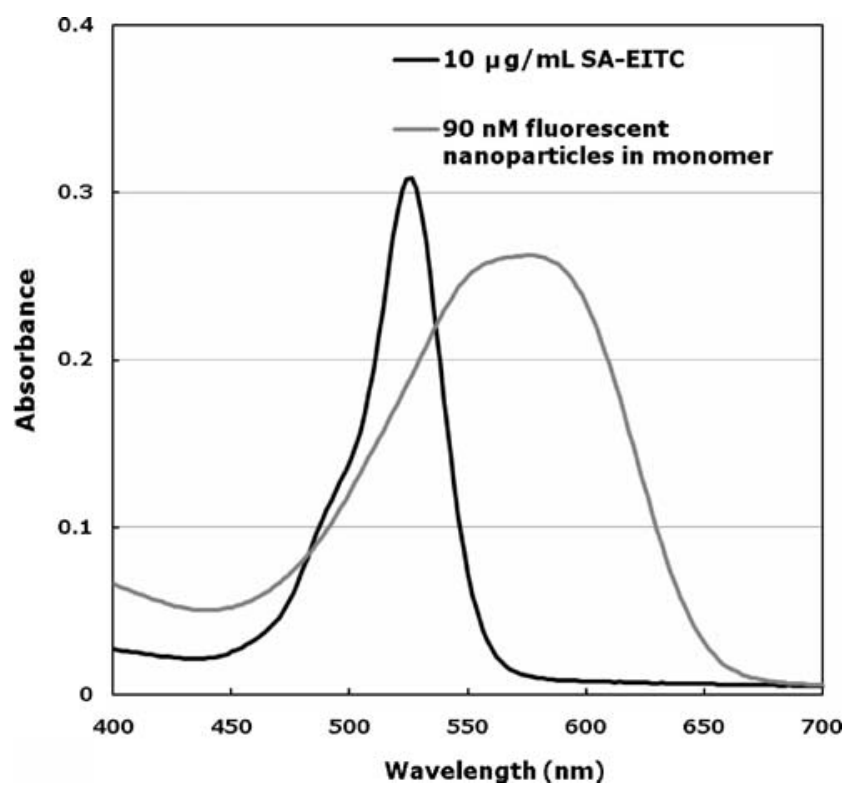

Fig. 5 Absorbance from SA-EITC product used to initiate the polymerization-based amplification and from fluorophores encapsulated in 20-nm polystyrene nanospheres and diluted to $90 \mathrm{nM}$ in PEGDA monomer formulation

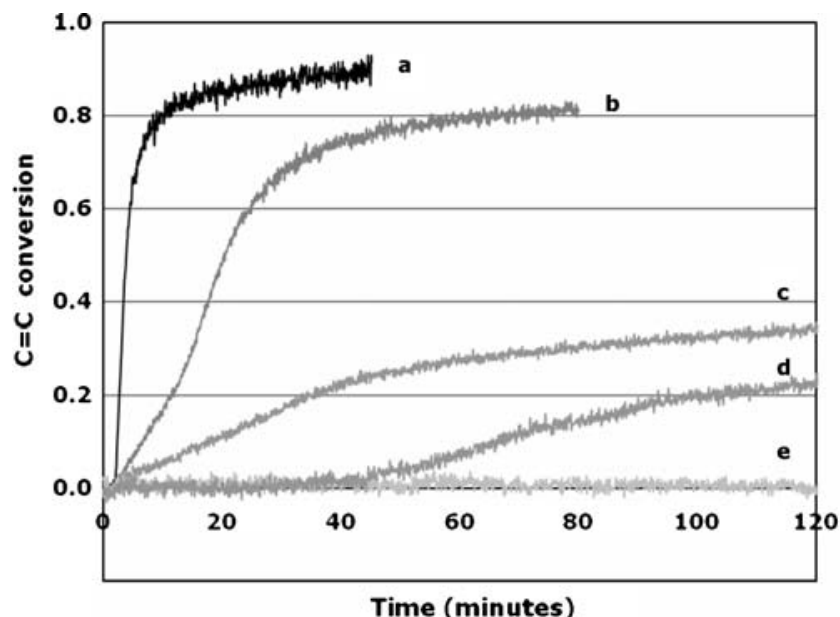

Fig. 6 FTIR plot of carbon-carbon double bond conversion of monomer (25\% (wt/vol) PEGDA, $225 \mathrm{mM}$ MDEA, $37.5 \mathrm{mM} \mathrm{1-}$ vinyl-2-pyrrolidinone, $\mathrm{pH}$ 9.0) versus time at different concentrations of fluorescent nanoparticles and photoinitiator using a 495-650-nm light source at $10 \mathrm{~mW} / \mathrm{cm}^{2} . a, 2 \mu \mathrm{M}$ eosin $(85 \mu \mathrm{g} / \mathrm{mL}$ SA-EITC), $0 \mathrm{nM}$ fluorescent nanoparticles. $b, 2 \mu \mathrm{M}$ eosin, $90 \mathrm{nM}$ fluorescent nanoparticles. $c, 2 \mu \mathrm{M}$ eosin, $480 \mathrm{nM}$ fluorescent nanoparticles. $d$, $0 \mu \mathrm{M}$ SA-EITC, $480 \mathrm{nM}$ fluorescent nanoparticles. $e, 0 \mu \mathrm{M}$ SA-EITC, $0 \mathrm{nM}$ fluorescent nanoparticles

fluorescent nanoparticles at nanomolar concentrations is thus considerably less efficient than initiation from SAEITC. This exposure window where polymerization occurs exclusively from SA-EITC allows for specificity in the amplification. Upon applying this system to a surfaceinitiated polymerization on dilution chips, polymerization at lower exposure will only occur due to the presence of SA-EITC from biotin-avidin binding. Unwanted, nonspecific initiation from the fluorescent nanospheres with bulk monomer does not occur until much longer exposure times. Thus, at lower exposures, the fluorescent monomer formulation is amenable to on-chip signal amplification in order to obtain amplified fluorescent signals.

Finally, amplification using monomer solutions containing nanomolar concentrations of Nile Red FluoSpheres from SA-ETIC-functionalized dilution chips were performed. At a diameter of $20 \mathrm{~nm}$, the nanospheres are either encapsulated or covalently linked into the PEGDA hydrogel matrix, and are not released during washing steps. Figure 7 details the increase in fluorescent signal from the amplification. The last seven columns corresponding to the seven higher-end biotin surface concentration points show an amplified, quantifiable fluorescent signal, and the highest gains are achieved with the nanoparticles used at $500 \mathrm{nM}$ concentrations in monomer. The number of encapsulated nanospheres and the corresponding fluorescent intensity increase monotonically with film thickness after amplification on dilution chips. This results in a large gradient in fluorescent signal ranging from 50 to $8000 \mathrm{Cy} 3$ 

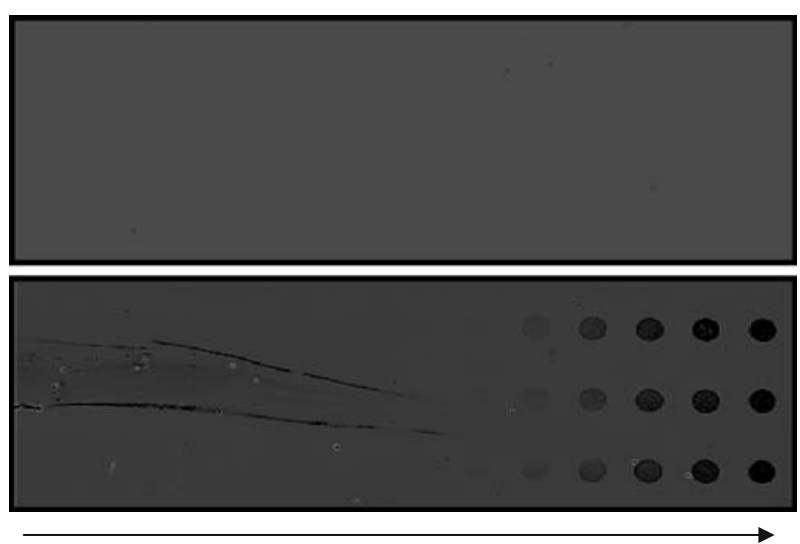

Increasing biotin-SA-EITC binding events $/ \mu \mathrm{m}^{2}$
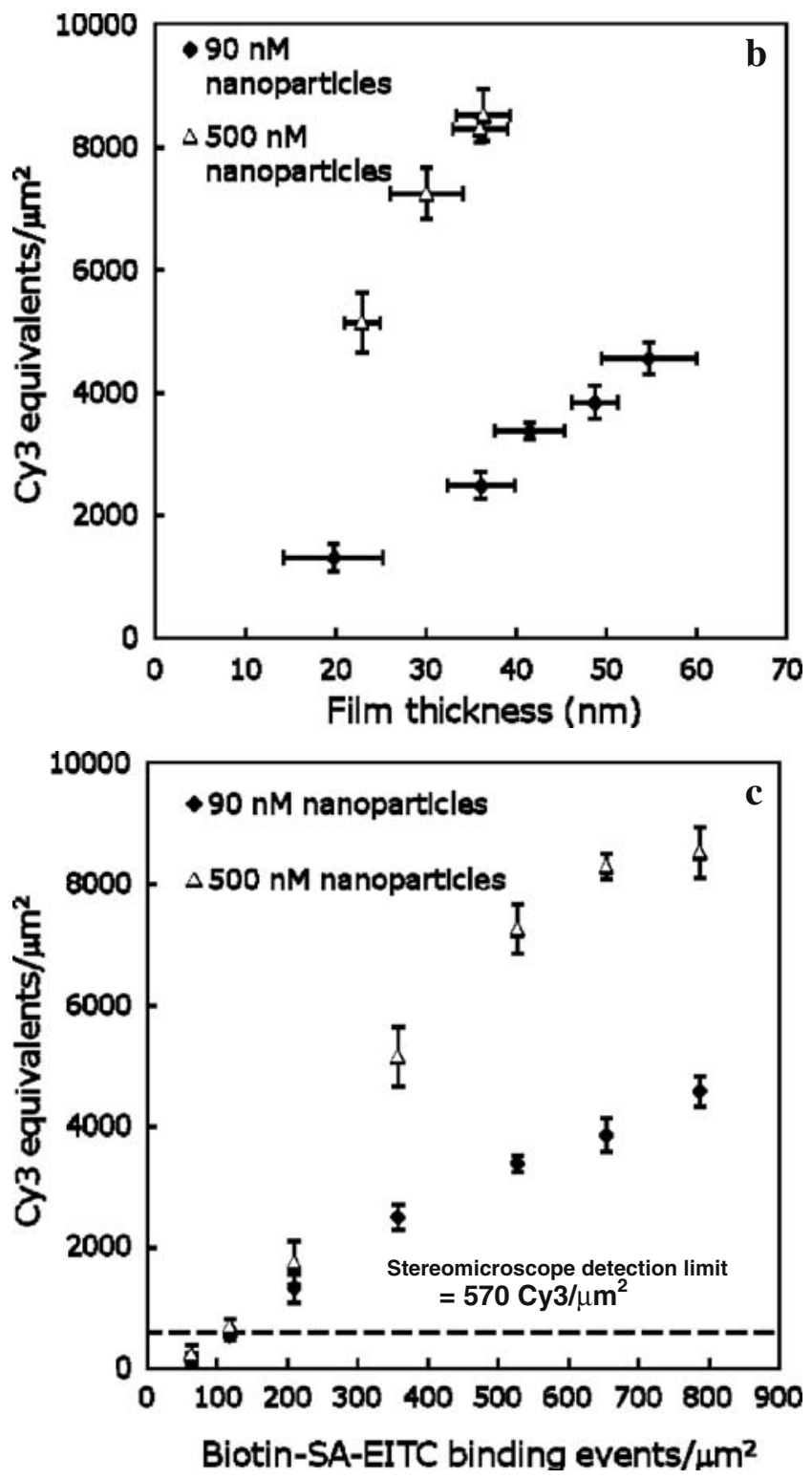

Fig. 7 a Stereomicroscope images of dilution chips before amplification (top) and after amplification (bottom) using fluorescent monomer solution (spots printed in triplicate). b Film thicknesses versus fluorescent signal generated from encapsulated fluorescent nanospheres after inclusion in monomer at 90 or $500 \mathrm{nM}$ concentrations. c Measured fluorophore densities versus biotin-SA-EITC binding events after polymerization-based amplification

fluors $/ \mu^{2}$, corresponding linearly to the number of biotinSA-EITC binding events generating the signal.

Moreover, the gain in fluorescent signal achieved through the amplification allows for the characterization of the biotinylated DNA concentrations using less sophisticated instrumentation amenable to point-of-care settings. Prior to amplification, SA-EITC-functionalized dilution chips were only detectable using a microarray scanner employing a PMT detector with low detection limits measured at $0.28 \mathrm{Cy} 3 \mathrm{fluors} / \mu^{2}$. After the amplification, the last six columns on the dilution chip were characterized using a stereomicroscope containing a CCD camera: a considerably less expensive, less sophisticated instrument with higher detection limits, measured at $570 \mathrm{Cy} 3$ fluors $/ \mu \mathrm{m}^{2}$.

The results presented represent an initial demonstration of the use of polymerization-based amplification to supply amplified, quantitative fluorescent signals useful for the measurement of DNA surface concentrations. Currently, the addition of the fluorescent nanoparticles decreases polymer film thicknesses and thus decreases the sensitivity of the assay as compared to amplification without their inclusion. This is consistent with the decrease in final conversions of bulk polymerizations with higher concentrations of nanoparticles noted in Fig. 6. Work is currently underway to obtain thicker polymer films containing higher concentrations of nanoparticles, allowing for higher gains in fluorescent signal and increased sensitivity with quantitative capability. Such advancements will further the use of polymerization-based amplification for quantitative applications.

\section{Conclusion}

Polymerization-based amplification has the ability to provide quantitative evaluation of genetic biomarkers over at least two orders of magnitude, as demonstrated through film thickness measurements correlating to the surface concentrations of biotinylated DNA targets. The photoinitiator surface density is a critical parameter in determining the amount of polymer growth observed from the surface, as this parameter can be manipulated depending on binding conditions to tune the amount of amplification generated from a given number of biotinylated targets localized on a biosensor surface. This amplification system is amenable to obtaining an amplified fluorescent response correlating with film 
thickness, enabling the evaluation of biomarker quantities with less expensive instrumentation.

The large, dynamic variation in film thicknesses and amplified fluorescent signal obtained from polymerizationbased amplification opens up many potential uses for this assay that were previously unachievable from only a positive/ negative response. For example, polymer growth arising from nonspecific biomolecule interactions can be characterized as background amplification such that only a deviation from this background becomes significant. This means that polymerization-based amplification can potentially be used in applications where both high specificity and sensitivity are critical, such as in a high-throughput SNP screening. Similarly, markers that become significant only when deviating from characterized background levels can also be analyzed. This is the case for the majority of proteomic biomarkers, and currently work is underway aimed at extending this assay to the detection of antigen-antibody binding.

Acknowledgements This work has been supported by the State of Colorado and the University of Colorado Technology Transfer Office. This work has also been supported by NIH 1R21 CA 127884. RRH would like to acknowledge the Graduate Assistantship in Areas of National Need Fellowship from the US Department of Education and the Teets Family Endowed Doctoral Fellowship in Nanotechnology for continued financial support of this work. HJA would like to acknowledge the National Science Foundation Research Fellowship for support of this work.

Open Access This article is distributed under the terms of the Creative Commons Attribution Noncommercial License which permits any noncommercial use, distribution, and reproduction in any medium, provided the original author(s) and source are credited.

\section{References}

1. Wittek M, Sturmer M, Doerr HW, Berger A (2007) Exp Rev Mol Diag 7:237-246
2. Ratcliff RM, Chang G, Kok T, Sloots TP (2007) Curr Issues Mol Biol 9:87-102

3. Diehl F, Li M, Dressman D, He Y, Shen D, Szabo S, Diaz LA Jr, Goodman SN, David KA, Juhl H, Kinzler KW, Vogelstein B (2005) Proc Natl Acad Sci USA 102:16368-16373

4. Diehl F, Diaz LA Jr (2007) Curr Opin Oncol 19:36-42

5. Ding C, Cantor CR (2004) J Biochem Mol Biol 37:1-10

6. Brena RM, Huang TH, Plass C (2006) J Mol Med 84:365-377

7. Bielas JH, Loeb LA (2005) Nature Methods 2:285-290

8. Kubista M, Andrade JM, Bengtsson M, Forootan A, Jonak J, Lind K, Sindelka R, Sjoback R, Sjogreen B, Strombom L, Stahlberg A, Zoric N (2006) Mol Aspects Med 27:95-125

9. Jarvius J, Melin J, Göransson J, Stenberg J, Fredriksson S, GonzalezRey C, Bertilsson S, Nilsson M (2006) Nature Methods 3:725-727

10. Li M, Diehl F, Dressman D, Vogelstein B, Kinzler KW (2006) Nature Methods 3:95-97

11. Tsongalis GJ (2006) Am J Clin Pathol 126:448-453

12. Sikes HD, Hansen RR, Johnson LM, Jenison R, Birks JW, Rowlen KL, Bowman CN (2007) Nature Materials 7:52-56

13. Hansen RR, Sikes HD, Bowman CN (2008) Biomacromolecules 9:355-362

14. Wilcheck M, Bayer EA, Livnah O (2006) Immunol Lett 103:27-32

15. Do JH, Choi DK (2007) Eng Life Sci 7:26-34

16. Pastinen T, Raitio M, Lindroos K, Tainola P, Peltonen L, Syvänen AC (2000) Genome Res 10:1031-1042

17. Hultin E, Kaller M, Ahmadian A, Lundeberg J (2005) Nucleic Acids Res 33:e48

18. Erdogan F, Kirchner R, Mann W, Ropers HH, Nuber UA (2001) Nucleic Acids Res 29:e36

19. Michel W, Mai T, Naiser T, Ott A (2007) Biophys J 92:999-1004

20. Park HG, Song JY, Park KH, Kim MH (2006) Chem Eng Sci 61:954-965

21. Andras SC, Power JB, Cocking EC, Davey MR (2001) Mol Biotech 19:29-44

22. Kizilel S, Pérez-Luna VH, Teymour F (2006) Macromol Theory Simul 15:686-700

23. Halperin A, Buhot A, Zhulina EB (2004) Biophys J 86:718-730

24. Peterson AW, Heaton RJ, Georgiadis RM (2001) Nucleic Acids Res 29:5163-5168

25. Epstein JR, Biran I, Walt DR (2002) Anal Chim Acta 469:3-36

26. Bally M, Halter M, Vörös J, Grandin HM (2006) Surf Interface Anal 38:1442-1458

27. Kizilel S, Sawardecker E, Teymour F, Pérez-Luna VH (2006) Biomaterials 27:1209-1215 